www.jmscr.igmpublication.org

Impact Factor 5.84

Index Copernicus Value: 83.27

ISSN (e)-2347-176x ISSN (p) 2455-0450

crossref DOI: https://dx.doi.org/10.18535/jmscr/v5i2.48

\title{
Rare Finding of Sinus Venosus type Atrial Septal Defect with Partial Anomalous Pulmonary Venous Connection presenting as Right Heart Failure and Pulmonary Hypertension in a young Adult
}

\author{
Authors \\ Dr Ashok Duggal ${ }^{1}$, Dr B.S.Bal ${ }^{2}$, Dr Rakesh Aggarwal ${ }^{3}$ \\ ${ }^{1}$ Associate Professor, department of Medicine, Government Medical College, Amritsar \\ ${ }^{2}$ Professor, Department of Medicine, Government Medical College, Amritsar \\ ${ }^{3}$ Assistant Professor, Department of Medicine, Government Medical College, Amritsar \\ Corresponding Author \\ Dr Ashik Duggal \\ 2229-C Block, Ranjit Avenue, Amritsar, Punjab
}

\begin{abstract}
Summary
Atrial septal defects can present with pulmonary arterial hypertension in adults. The age at which symptoms appear is highly variable and is not exclusively related to the size of the shunt. Consequently, patients with ASD are at risk for developing atrial dysrhythmias with an inherent risk of thromboembolic complications. Careful assessment for these defects, and their associated pulmonary venous anomalies in patients with enlarged central pulmonary vessels, may be beneficial as surgical repair of these defects can improve outcomes. Sinus venosus defect may carry a worse prognosis than other forms of ASD and may need to be treated at a younger age with surgical closure.
\end{abstract}

\section{Introduction}

Sinus venosus atrial septal defect (SVASD), originally described in 1858, encompasses approximately $4 \%$ to $11 \%$ of atrial septal defects (ASDs) $)^{1,2}$ The typical malformation is an interatrial communication caused by deficiency of common wall between superior venacava (SVC) and right sided pulmonary vein., ${ }^{2,3}$ SVASD is commonly associated with anomalous pulmonary venous connection (APVC) of some or all the pulmonary veins. ${ }^{3,4}$ The anomalous pulmonary vein usually right upper or middle pulmonary vein can either override interatrial septum (anomalous drainage) or can drain separately into superior venacava (true anomalous connection). The increased pulmonary venous flow associated with partial anomalous pulmonary venous connection (PAPVC) can eventually lead to dyspnoea on exertion, atrial arrythmias, pulmonary hypertension (PHT) and right sided heart failure (RHF). Even less commonly, the discovery of cyanosis may lead to the diagnosis of an intraatrial communication, cyanosis being more common in inferior sinus venosus defects.

We report an unusual case of 32 year old patient presenting with PHT and RHF who was found to have PAPVC involving right upper pulmonary vein with SVASD.

\section{Case report}

A 32 year old male presented to our hospital with dyspnoea on exertion (NYHA grade II) 


\section{JMSCR Vol||05||Issue||02||Pages 17502-17508||February}

accompanied by chest pain and palpitation but no history of orthopnea and paroxysmal nocturnal dyspnoea (PND). There was history of 2 episodes of mild hemoptysis 1yr back.

On initial presentation, notable findings included $\mathrm{HR}$ of $86 \mathrm{bpm}$; BP of 100/70 mm of $\mathrm{Hg}$; RR 18/min; central cyanosis, clubbing; bipedal edema; RV heave and apex beat in $5^{\text {th }}$ ICS $1 \mathrm{~cm}$ outside left MCL. Cardiac auscultation revealed $\mathrm{P}_{2}$ loud along with grade III ejection systolic murmur in pulmonary area; pansystolic murmur which increased on inspiration in tricuspid area.

An electrocardiogram depicted right axis deviation and incomplete RBBB along with 'p'pulmonale.

The chest radiograph demonstrates increased central pulmonary vascularity ("pulmonary arterial hypertension" pattern) without evidence of pulmonary oedema; the cardiac silhouette was moderate enlarged, primarily from right heart enlargement.

Transthoracic echocardiography (TTE) demonstrated dilated right atrium (RA) and right ventricle (RV); sinus venosus type of ASD with anomalous drainage of right upper pulmonary vein into RA; mild tricuspid regurgitation (TR) with peak regurgitant velocity of $-5.44 \mathrm{~m} / \mathrm{sec}$; hence pressure gradient of $118.5 \mathrm{~mm} \mathrm{Hg}$ between RV-RA during systole and pulmonary artery systolic pressure (PASP) of $128.5 \mathrm{~mm}$ of $\mathrm{Hg}$ depicting severe PHT.

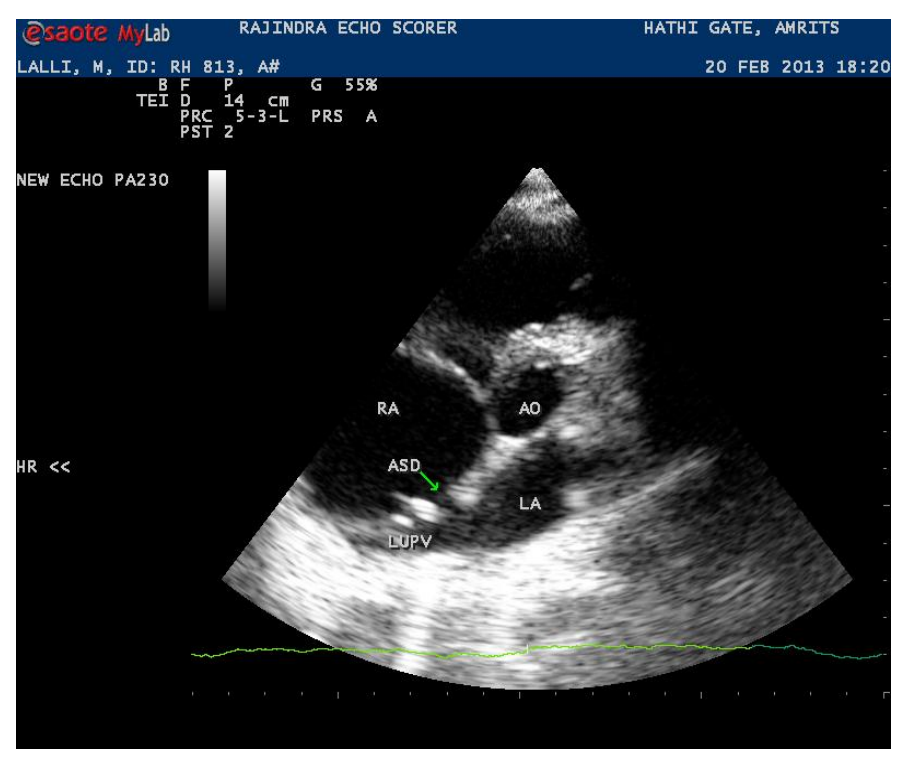

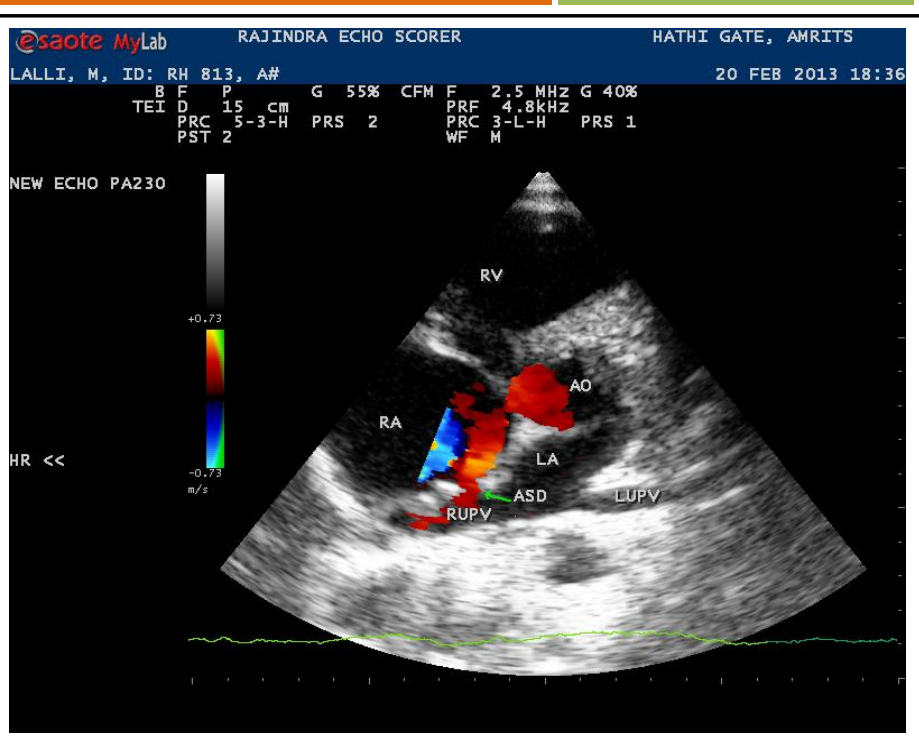
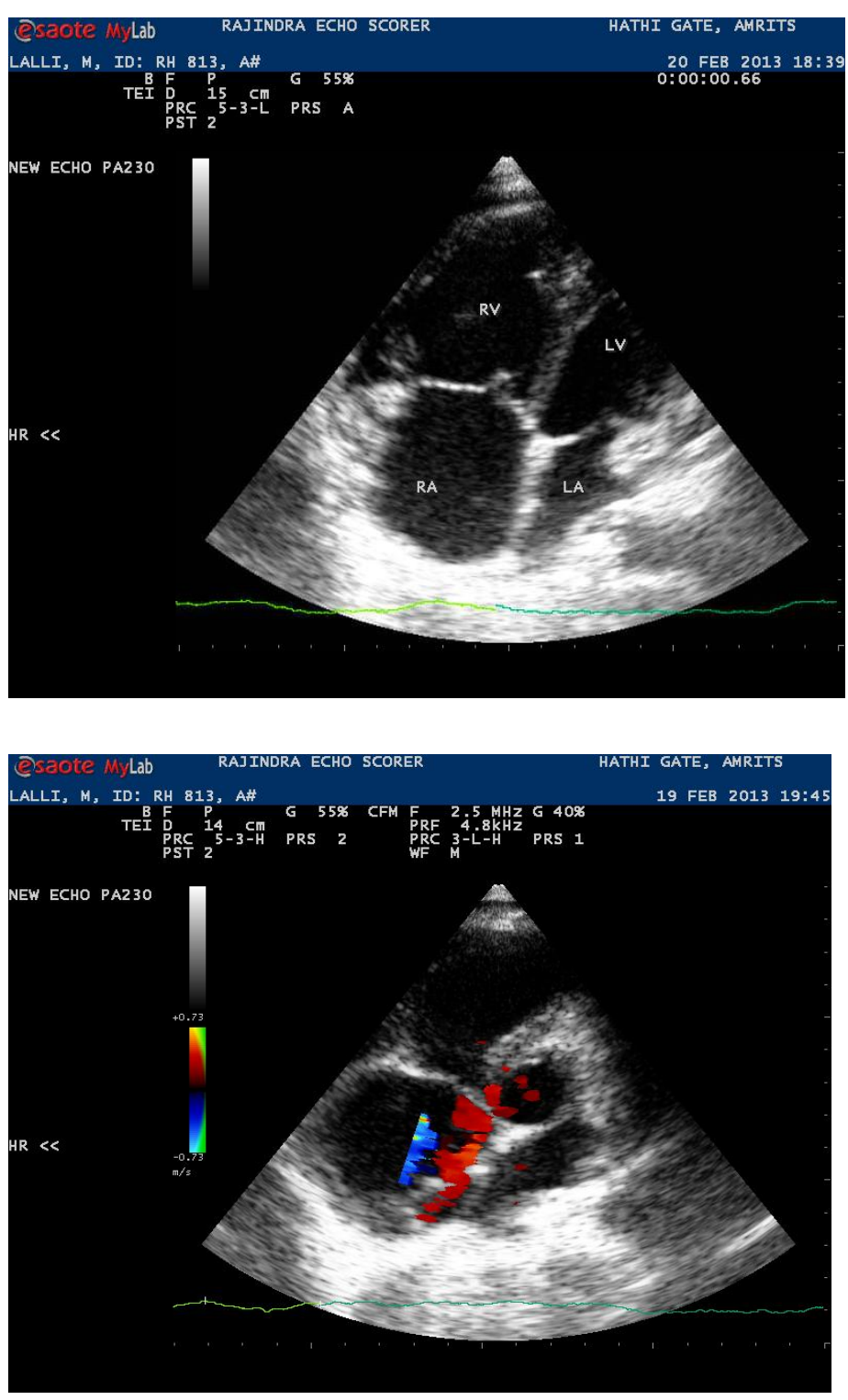

\section{Discussion}

During normal embryonic development, the right horn of the sinus venosus encompasses the right superior vena cava (SVC) and inferior vena cava 
(IVC). If abnormal resorption of the sinus venosus occurs, an atrial septal defect results near the orifice of either the SVC or IVC. Sinus venosus atrial septal defects are congenital lesions present at birth. The age at presentation depends on the size of the left-to-right shunt.

Atrial septal defects account for about $10-15 \%$ of all congenital cardiac anomalies and are the most common congenital cardiac lesion presenting in adults. Sinus venosus atrial septal defects account for only $10 \%$ of atrial septal defects. The remaining atrial septal defects are ostium secundum type (70\%), ostium primum type $(20 \%)$, and unroofed coronary sinus, or coronary sinus septal defects, $(<1 \%)$.

The more common sinus venosus type defect (often referred to as the "usual type") occurs in the upper atrial septum and is contiguous with the superior vena cava (SVC). The lesion is rostral and posterior to the fossa ovalis (where secundum type defects occur) and is separate from it. It is almost always associated with anomalous pulmonary venous drainage of the right upper pulmonary vein into the SVC. Less commonly, the defect may occur at the junction of the right atrium and inferior vena cava (IVC) and be associated with anomalous connection of the right lower pulmonary vein to the IVC. Rarely, sinus venosus defects occur posterior to the fossa ovalis without bordering the SVC or IVC. The predominant hemodynamic consequence is a leftto-right shunt through the defect.

Atrial septal defects in infancy are usually asymptomatic. They are usually detected by echocardiography while undergoing a cardiac evaluation. Sinus venosus atrial septal defects are diagnosed upon detection of a murmur, a split second heart sound, and/or right heart enlargement on ECG in the usually asymptomatic patient.

Symptoms of atrial septal defects are typically a function of the size of the associated shunt. As many as $60 \%$ of apparently asymptomatic patients may have easy fatigability and dyspnoea. Such symptoms usually indicate a relatively large shunt. Presence of cyanosis in atrial septal defect without pulmonary hypertention, a platypnea-orthodeoxya syndrome should be investigated to exclude coexisting clinical conditions. The presence of cyanosis usually suggests advanced pulmonary hypertension, but it may result from associated anomalies such as pulmonary stenosis or even from a prominent Eustachian valve directing inferior vena cava flow to the left atrium through an ASD (particularly of the inferior sinus venosus type). In our case it was advanced $\mathrm{PH}$, as patients with sinus venosus defect have higher pulmonary pressures and resistances and develop these complications at younger age than patients with atrial septal defects.

Adults may have "gracile habitus." (thin for their height) and may not come to medical attention until symptoms occur; usually presents with arrhythmias, dyspnoea, and a decrease in exercise tolerance. A cardiac murmur usually a grade 2$3 / 6$ systolic ejection murmur secondary to increased pulmonary artery blood flow is heard over the left sternal border. A prominent right ventricular impulse may also be noted along the left sternal border. A diastolic flow murmur may be present at the left lower sternal border with or without systolic murmrmur in tricuspid area and is indicative of a large left-to-right shunt.

The second heart sound is widely split and may be fixed or may vary little with respiration. The pulmonic component of the second heart sound is usually normal in intensity but may increase in intensity if pulmonary hypertension is present.

Atrial septal defects occur as associated anomalies in many major complex congenital lesions but sinus venosus atrial septal defects occur more often as an isolated abnormality. Other abnormalities may exacerbate an atrial septal defect. For instance, systemic hypertension in an adult with a sinus venosus atrial septal defect may result in left ventricular hypertrophy and reduce left ventricular compliance, which, in turn, exacerbates the atrial level left-to-right shunt. Mitral stenosis, which is either congenital or acquired, may also exacerbate the atrial level leftto-right shunt. 


\section{Workup includes}

Chest radiography which may show

- Prominent right atrium. Right heart dilation is better appreciated in lateral films.

- Increased heart size.

- Prominent main pulmonary artery and pulmonary vascularity

- A small aortic knuckle is characteristic, which reflects a chronically low systemic cardiac output state, because increased pulmonary flow in these patients occurs at the expense of reduced systemic flow.

\section{Echocardiography}

- Echocardiography (ECHO) is the diagnostic modality of choice which reveals atrial septal defect including pulmonary vein connections.

- TTE with colour flow Doppler reveals the position and size of the defect and the presence of anomalous pulmonary venous drainage in majority of patients.

In addition reveals the left-to-right (or right-to-left) direction of flow and the degree of right ventricular overload and also identifies associated anomalies

TEE may be helpful in imaging the defect and pulmonary vein connections in patients with difficult TT windows especially in children, obese and in older.

\section{Electrocardiogram}

- Right ventricular hypertrophy predominates, with a lengthened PR interval and incomplete right bundle branch block (small rSR').

- P wave morphology of atrial enlargement. Inverted $\mathrm{P}$ waves in the inferior leads suggest an absent or deficient sinus node, as may be seen in a sinus venosus defect. ${ }^{5,6}$

\section{Cardiac MRI/MRA}

In the current era, cardiac magnetic resonance angiography (MRA)/MRI may be alternatively used to complete the diagnostic information needed prior to surgery
- Atrial septal defect size and location are shown.

- Excellent delineation of individual pulmonary vein connections can be identified.

- Right ventricle enlargement and indexing to body surface area (BSA) is available if helpful.

- Flow-quantification may also be performed

Cardiac catheterization is usually not required in the preoperative assessment of patients with sinus venosus atrial septal defect, but it may be considered in the following circumstances

- In any child in whom associated lesions are suspected or in whom pulmonary hypertension is suspected, catheterization is performed to measure pulmonary artery pressure and, if pulmonary resistance is elevated, the response to pulmonary vasodilators.

- Adults who have the potential for associated coronary atherosclerotic lesions should undergo catheterization to exclude these abnormalities before surgical repair of the sinus venosus atrial septal defect.

Patients with pulmonary hypertension and advanced pulmonary vascular obstructive disease may exhibit histologic changes similar to those seen in pulmonary vascular disease. Specifically, these include intimal and medial hypertrophy and, in more advanced lesions, luminal occlusion

\section{Treatment}

Atrial septal defects affect females more often than males(FM ratio is $2: 1)$. No difference in outcome is associated with sex.

\section{Medical}

Medical care of sinus venosus atrial septal defect (ASD) is primarily supportive and is not required for asymptomatic patients. Patients presenting in heart failure should be stabilized with diuretics and inotropic support in anticipation of elective repair. There are some ASD patients in whom 
repair is not possible because of high pulmonary arterial pressures and pulmonary vascular resistance, usually when the left-to-right shunt is reversed. There have been case reports of such patients being managed with intravenous epoprostenol or oral bosentan with such success that ASD closure subsequently became possible. ${ }^{7,8}$

\section{Surgical}

Sinus venosus defects do not close spontaneously. Surgical correction is the mainstay of therapy. Asymptomatic children generally undergo repair when aged 3-5 years. Cardiac size rapidly regresses after surgery, and the functional result is excellent

Adults with left-to-right shunts greater than 1.52:1 benefit from surgical closure. In cases of repair during adulthood, life expectancy may be decreased despite successful repair. Surgical morbidity rates are related to early postoperative pericardial effusion, early postoperative pulmonary venous or systemic venous obstruction, and supraventricular arrhythmias.

After age 20 years, the mortality rate is approximately $5 \%$ per decade with $90 \%$ of patients dead by age 60 years. These patients present with an increase in left-to-right shunting and occasionally with congestive heart failure with pulmonary hypertension in the fourth to sixth decades of life. Late problems in untreated patients also include the risk of paradoxical embolus as well as atrial fibrillation, pulmonary hypertension, and right heart failure.

Patients with significant pulmonary hypertension and elevated pulmonary vascular resistance unresponsive to pulmonary vasodilator therapy (eg, oxygen, nitric oxide, calcium channel blockers,) may not be good candidates for surgical repair. Pulmonary arterial hypertension may be too advanced, contraindicating ASD closure; the ASD may be physiologically needed by the patient (eg, as a "pop-off" valve in a patient with severe pulmonary hypertension). Such patients are often cyanotic at rest and become more cyanosed during exercise. Such patients may develop acute right ventricular failure if their heart no longer has the ability to shunt right to left at the atrial communication in response to increases in pulmonary vascular resistance.

\section{An atrial septal defect was the first lesion repaired using cardiopulmonary bypass in 1954 by John Gibbon, MD, at the Mayo Clinic.}

Repair is performed mostly through a standard median sternotomy. However, cosmetic incisions may also be used, such as partial sternotomies, small right anterior thoracotomies, and inframammary incisions. All approaches require the use of cardiopulmonary bypass for closure of the atrial septal defect.

Transcatheter occlusion devices are not indicated (at present) for the closure of sinus venosus atrial septal defects because of the position of the defect and because of the lack of surrounding tissue adequate to place such an occlusion device. Additionally, it does not achieve redirection of the anomalous right pulmonary venous flow to the left atrium and may obstruct SVC flow.

Repair of the sinus venosus atrial septal defect is more complex than repair of the average secundum atrial septal defect. A patch (synthetic material or pericardium) is used to redirect blood flow from the right superior pulmonary vein into the left atrium. This effectively closes the interatrial communication while also correcting the anomalous pulmonary venous drainage. Sometimes, to avoid creating superior vena cava (SVC) obstruction, a patch is placed on the anterior surface of the SVC. Care is taken to avoid injuring the nearby sinus node. Ligation of the azygous vein may also be required to eliminate its drainage into the left atrium and to prevent the resulting residual right-to-left shunt.When the location of the anomalous venous drainage is in the high SVC and is far from the atrial-caval junction, a different surgical approach can be used to decrease the probability of caval stenosis or pulmonary vein stenosis

Complications following surgery include pulmonary venous obstruction, arrhythmias (AF,AFL,SVT), pericardial effusion, atrial baffle 
leak, SVC syndrome and sinus node dysfunction. If the baffle directing pulmonary venous blood to the left atrium is not placed correctly, it may obstruct pulmonary venous drainage. If the baffle bulges into the SVC, it may obstruct SVC inflow, necessitating the placement of an augmentation patch on the anterior surface of the SVC and right atrial junction. All reported series have demonstrated excellent results with little or no pulmonary venous or SVC stenosis. In addition, concern for injury to the conduction system or sinus node have not been observed to date.

Postoperatively, Echocardiography is done to evaluate the repair for evidence of residual shunting, superior vena cava (SVC) or pulmonary vein obstruction, pericardial effusion, and ventricular function. Postoperative narrowing of the SVC and sinus node dysfunction screening should be carried out which are usually late complications. Most physicians would follow up adult patients for at least 1 year, or longer if a particularly large device was deployed.

As far aspirin or other anticoagulation regimens are concerned some surgeons prescribe these regimens for several weeks (low dose aspirin and clopidogrel usually precribed for at least 3 months) due to use of prosthetic patch to close the defect but long-term anticoagulation is not indicated. Antibiotic prophylaxis is not required in patients who have had atrial septal defects repaired.

\section{Prognosis}

Excellent surgical results with a mortality rate near $0 \%$ can be expected. This is particularly true in patients who undergo repair when younger than 15 years. Surgical repair in the first 2 decades of life is associated with a mortality rate near zero. Life expectancy approaches that of the general population if the defect is repaired during this time but repair delayed until the third decade of life is associated with a decrease in life expectancy. Surgical repair of ASD, including sinus venosus defect, in patients over 40 years of age, increases long-term survival and decreases the incidence of heart failure ${ }^{9,10}$. Untreated atrial septal defects on the other hand are associated with a significantly shortened life expectancy

\section{Conclusion}

Sizeable ASDs with right heart dilation are associated with important age-related morbidity and mortality.Current evidence would suggest that all types of ASDs with right heart dilation should be considered for timely closure once the diagnosis is established, irrespective of age. Functional improvement is expected irrespective of age at repair, but postoperative atrial fibrillation appears to be related to older age at operation. Sinus venosus defect may carry a worse prognosis thus they should be managed differently than patients with "simple" atrial septal defects.

\section{References}

1. Peacock TB, Malformations of the heart: Peacock TB, ed. On malformations of the human heart: with original cases. London, UK: John Churchill; 1858: 11-102

2. Oliver JM, Gallego P, Gonzalez A, Domingue ZFJ, Aroca A, Mesa JM. Sinus Venosus Syndrome; Atrial septal defect or anomalous venous connection? A multiplane transesophageal approach. Heart 2002; 88: 634-638

3. Vaan Praagh, Carrera ME, Sanders SP, Mayor JE, Van Praagh R. sinus venosus defects; unroofing of right pulmonary veins: Anatomic and echocardiographic findings and surgical treatment. Am Heart J, 1994; 128: 365-379

4. Kirklin JW, Barrratt Boyes BG, eds. Cardiac Surgery: Diagnostic Criteria, Natural History, Techniques, Results and Indications. $2^{\text {nd }}$ ed. New York, NY: Churchill Livingstone; 1993; 1: 609-644

5. Jost CH, Connolly HM, Danielson GK, Bailey KR, Schaff HV, Shen WK, Warnes CA, Seward JB, Puga FJ, Tajik AJ. Sinus venosus atrial septal defect: long-term postoperative outcome for 115 
patients. Circulation. 2005; 112:1953-

1958.

6. Davia JE, Cheitlin MD, Bedynek JL. Sinus venosus atrial septal defect: analysis of fifty cases. Am Heart J. 1973; 85: 177185.

7. Frost AE, Quinones MA, Zoghbi WA, Noon GP. Reversal of pulmonary hypertension and subsequent repair of atrial septal defect after treatment with continuous intravenous epoprostenol. $J$ Heart Lung Transplant. 2005;24: 501503.

8. Schwerzmann M, Zafar M, McLaughlin PR, Chamberlain DW, Webb G, Granton J. Atrial septal defect closure in a patient with "irreversible" pulmonary hypertensive arteriopathy. Int J Cardiol. 2006; 110: 104-107.

9. Agrawal SK, Khanna SK, Tampe D. Sinus venosis atrial septal defects: Surgical follow-up. European J Cardiothoracic Surg 1997; 11(3): 455-7.

10. Konstantinides $S$ et. al. A comparison of surgical and medical therapy for atrial septal defects in adults. NEJM 1995; 333(8): 469-73. 